\title{
Identification of surface anion antisite defects in (110) surfaces of III-V semiconductors
}

Cite as: Appl. Phys. Lett. 79, 2877 (2001); https://doi.org/10.1063/1.1408906

Submitted: 24 April 2001 . Accepted: 31 July 2001 . Published Online: 23 October 2001

Ph. Ebert, P. Quadbeck, K. Urban, B. Henninger, K. Horn, G. Schwarz, J. Neugebauer, and M. Scheffler

ARTICLES YOU MAY BE INTERESTED IN

Band parameters for III-V compound semiconductors and their alloys

Journal of Applied Physics 89, 5815 (2001); https://doi.org/10.1063/1.1368156

Low-temperature growth of SiCAIN films of high hardness on Si(111) substrates

Applied Physics Letters 79, 2880 (2001); https://doi.org/10.1063/1.1413723

Native point defects in low-temperature-grown GaAs

Applied Physics Letters 67, 279 (1995); https://doi.org/10.1063/1.114782
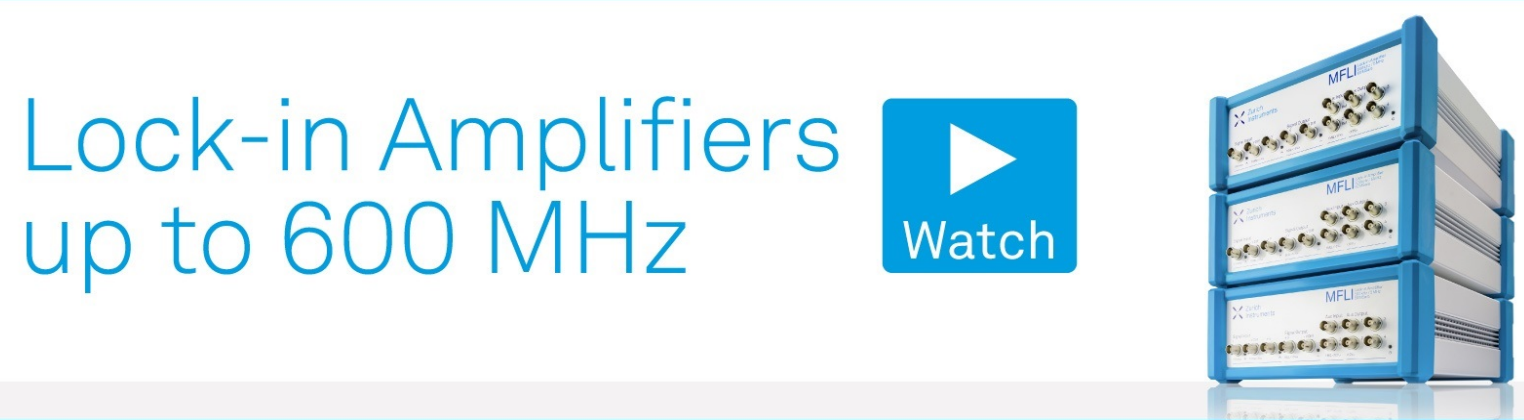


\title{
Identification of surface anion antisite defects in (110) surfaces of III-V semiconductors
}

\author{
Ph. Ebert, ${ }^{\text {a) }}$ P. Quadbeck, and K. Urban \\ Institut für Festkörperforschung, Forschungszentrum Jülich GmbH, 52425 Jülich, Germany \\ B. Henninger, K. Horn, G. Schwarz, J. Neugebauer, and M. Scheffler \\ Fritz-Haber-Institut der Max-Planck-Gesellschaft, Faradayweg 4-6, 14195 Berlin, Germany
}

(Received 24 April 2001; accepted for publication 31 July 2001)

\begin{abstract}
We identify surface anion antisite defects in (110) surfaces of GaAs, GaP, and InP using scanning tunneling microscopy combined with density-functional theory calculations. In contrast to subsurface arsenic antisite defects, surface antisite defects are electrically inactive and have a very localized defect state which gives rise to a distinct feature in scanning tunneling microscopy images.

(c) 2001 American Institute of Physics. [DOI: 10.1063/1.1408906]
\end{abstract}

Low-temperature grown GaAs layers are very promising for device applications, because of their exceptional properties, such as a fast absorption recovery time or high resistivities. These properties are closely connected with the incorporation of excess arsenic at low growth temperatures, which leads to the formation of a high concentration of defects, whose nature has long been debated. Cross sectional scanning tunneling microscopy (STM) showed directly that the defects formed by the incorporation of excess arsenic are isolated arsenic antisite $\left(\mathrm{As}_{\mathrm{Ga}}\right)$ defects with no complex formation and that the antisite defects give rise to an intense band of midgap states. ${ }^{1,2}$ One of the most striking features revealed by the STM images has been the spatially extended local density of states of the defects which includes satellite features. ${ }^{1,3}$ Based on the symmetry and intensity of the local density of states as imaged by STM, arsenic antisite defects in different subsurface layers were distinguished.

In this letter, we report the identification of anion antisite defects in the surface layer of (110) surfaces of GaAs, GaP, and InP using STM experiments and density-functional theory (DFT) calculations. We demonstrate that the anion antisite defect in the surface layer of (110) surfaces has a very localized density of states with no satellite peaks, in contrast to the extended density of states of subsurface antisite defects as reported in Ref. 1. Furthermore, the surface anion antisite defects are electrically inactive and thus cannot induce a Fermi-level pinning unlike bulk antisite defects. The surface anion antisite defects have essentially the same properties on all three investigated materials.

For the investigation of surface antisite defects, samples from different $n$-doped liquid encapsulated Czochralski grown $\mathrm{GaAs}, \mathrm{GaP}$, and InP crystals with carrier concentration of $(1.5-3.5) \times 10^{18}$ (Te-doped GaAs), $1 \times 10^{18}(\mathrm{Si}-$ doped GaAs), $(5.6-6.0) \times 10^{17} \quad($ S-doped GaP), and $(0.9-1.8) \times 10^{18}($ Sn-doped $\mathrm{InP}) \mathrm{cm}^{3}$ were cleaved in ultrahigh vacuum. Directly after cleavage, the surfaces were examined by STM in their as-grown state without breaking of the vacuum.

Figure 1 shows an overview of such a $\mathrm{GaP}(110)$ cleav-

${ }^{\text {a)}}$ Electronic mail: p.ebert@fz-juelich.de age surface. The constant-current STM image exhibits rows of occupied dangling bonds localized above the $\mathrm{P}$ atoms of the $1 \times 1$ surface structure. ${ }^{4,5}$ In addition to the regularly spaced dangling bonds, we observe additional density-ofstates maxima between the rows marked by arrows in Fig. 1 . The lack of any apparent height change or long-range voltage dependent contrast around the features in large scale STM images indicates that the underlying defects are electrically uncharged on all investigated $n$-doped surfaces. ${ }^{6,7} \mathrm{We}$ also observed these defects in a neutral charge state on GaAs surfaces pinned by high concentrations of steps, indicating that the defects are also uncharged for Fermi-level positions near midgap.

Figures 2(a) and 2(b) show high resolution STM images of the occupied and empty density of states of such a defect on the $n$-doped GaAs(110) surface. The additional maxima in the occupied density of states of the defect can be clearly recognized [Fig. 2(a)]. In addition, the four surrounding occupied dangling bonds exhibit a change in their morphology at low negative voltages. They are shifted towards the additional maximum and exhibit a rotation of about $45^{\circ}$ relative to the direction of the rows. Figures 3(a) and 3(c) show these

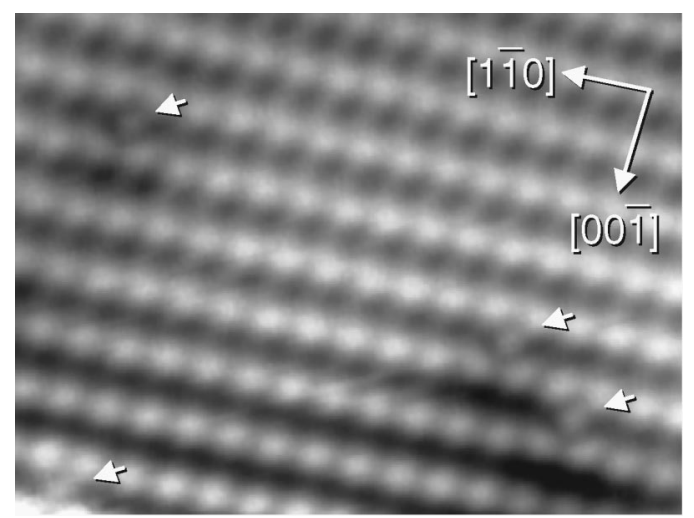

FIG. 1. STM image of a $5.5 \times 4.5 \mathrm{~nm}^{2}$ area of the $n$-doped GaP(110) cleavage surface. Four $\mathrm{P}_{\mathrm{Ga}}$ antisite defects in the surface layer are marked by arrows. The image has been acquired at $-2.7 \mathrm{~V}$ tunneling voltage and 0.4 nA current. 

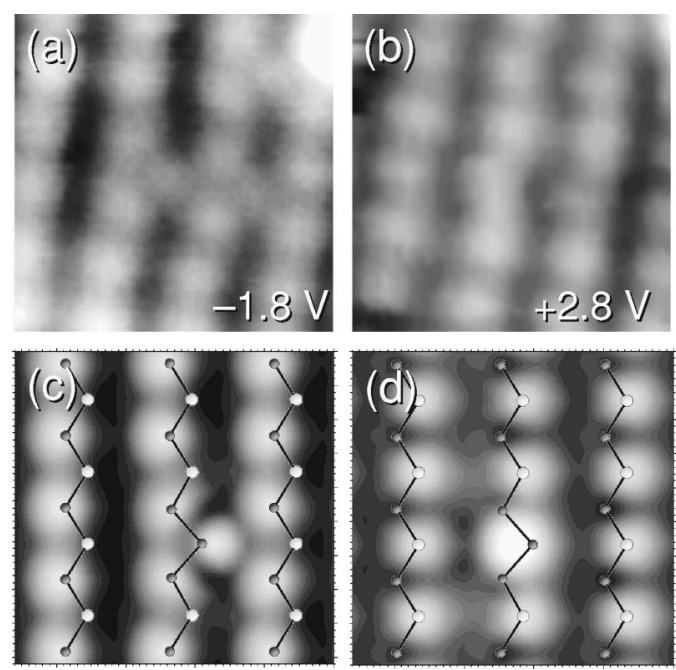

FIG. 2. Measured and calculated STM images of a surface arsenic antisite defect $\left(\mathrm{As}_{\mathrm{Ga}}\right)$ in $n$-doped $\mathrm{GaAs}(110)$ surfaces. (a) Filled states STM image at $-1.8 \mathrm{~V}$, (b) empty states STM image at $+2.8 \mathrm{~V}$, (c) calculated filled state image including states from the VBM up to VBM $-0.7 \mathrm{eV}$, and (d) calculated empty state image including all unoccupied states below VBM $+2.0 \mathrm{eV}$. The charge density in the calculated images ranges from 1 $\times 10^{-5}$ to $1 \times 10^{-4} \AA^{-3}$ (c) and from $1 \times 10^{-4}$ to $5 \times 10^{-4} \AA^{-3}$ (d).

effects for analogous defects on (110) cleavage surfaces of InP and $\mathrm{GaP}$. In contrast to the occupied states, the empty states exhibit much more subtle changes [Fig. 2(b)]. Their density-of-states images reveal that the empty dangling bond localized at the defect center is slightly displaced in the [001] direction.

We also observe a pronounced voltage dependence of the occupied STM images. With increasing magnitude of voltage, the additional features in the occupied density-ofstates images vanish, such that at high voltages [Figs. 3(b) and 3(d)], the defect is hardly visible. In addition, the neighboring occupied dangling bonds exhibit no detectable displacements at high bias voltages for all materials investigated, in contrast to the observation at lower voltages. Therefore, at higher bias the surface appears to be apparently free of defects, even if the STM images acquired at low
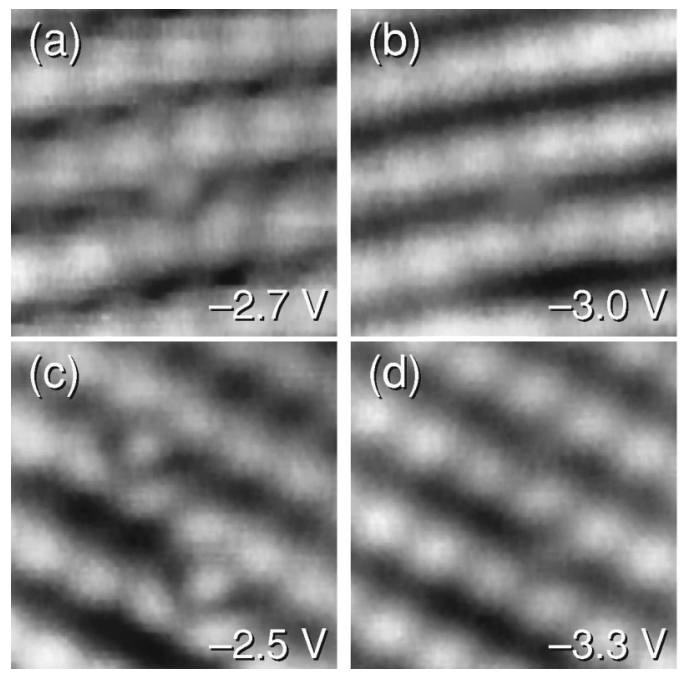

FIG. 3. Voltage dependence of the STM images of phosphorus antisite defects in $n$-doped $\mathrm{GaP}(110)\left[\mathrm{P}_{\mathrm{Ga}}\right.$; (a) and (b)] and in $\operatorname{InP}(110)\left[\mathrm{P}_{\mathrm{In}}\right.$; (c) and (d)] surfaces is shown. The voltages are indicated in the individual images. voltages clearly demonstrate the presence of the defects. The voltage dependence also suggests that the additional density of states observed in Figs. 2(a), 3(a), and 3(c) corresponds directly to an occupied localized defect state in the bandgap or in the top-most part of the valence band, because it is only imaged at low magnitudes of negative sample voltage, where the STM image samples the density of states close to the valence band maximum (VBM). At larger voltages, the density of states localized at the occupied dangling and back bonds dominate the contrast of the STM images. ${ }^{5}$

At this stage, we focus on the identification of the nature of the defect. Since we cannot unambiguously identify the defect on basis of the STM images alone, we performed a systematic DFT study of all possible intrinsic point defects (vacancies, interstitials, and antisities) on the (110) surface of III-V semiconductors. Details of the calculational method used are described in Refs. 8 and 9. We used a supercell with a $2 \times 4$ periodicity in the [001] and [ $\overline{1} 10]$ directions consisting of six layers. This is large enough to describe the defect properly, since tests showed that the defect formation energies change typically by less than $10 \mathrm{meV}$ when further increasing the size of the supercell. The calculated equilibrium position of $\mathrm{As}_{\mathrm{Ga}}$ is shifted by $0.5 \AA$ in the [001] and the [110] direction as compared to the surface cations and is thus locally lifting the surface buckling between surface arsenic and gallium. In order to directly compare the calculations with the STM images, we have calculated simulations of STM images from the local density of states for all surface defects employing a Tersoff-Hamann type of analysis. ${ }^{8,10}$ In these calculations, the supercell was increased to a $3 \times 4$ periodicity in order to resolve the signature of the isolated defect on the neighboring zigzag rows. The simulations show that only the surface $\mathrm{As}_{\mathrm{Ga}}$ defect yields good agreement with the experimental data. Thus, we can exclude all other defects (vacancies, interstitials, cation antisite defects, and all subsurface defects). On the basis of the experimental STM images, we can also rule out adatoms and dopant atoms, which have a distinctively different contrast than the defects studied here. ${ }^{11}$

Furthermore, the calculations show that the surface antisite defect has no charge transfer levels or defect states in the band gap. Thus, surface anion antisite defects are electrically neutral for all Fermi level positions and electrically inactive, in agreement with the observation of uncharged defects by STM. The defect-related resonance closest to the top of the VBM is found at VBM-0.6 eV and thus below the occupied $A_{5}$ surface band in agreement with recent tight-binding calculations. ${ }^{3}$ The electronic structure of the surface antisite is very much different from that of bulk antisite defects which are double donors ${ }^{12,13}$ with a defect level of $A_{1}$ symmetry in the middle of the band gap. ${ }^{14}$ Note that the absence of charges and defect levels in the band gap infer that surface anion antisite defects do not affect the position of the Fermi level at the surface unlike anion vacancies. ${ }^{15}$

Figures 2(c) and 2(d) show the simulated occupied and empty state STM images of the surface $\mathrm{As}_{\mathrm{Ga}}$ defect, respectively. We show the local density of states in energy intervals comparable to those probed by the STM at the specified voltages applied to the sample. The overall similarity of the experimental STM images and the simulation is clearly 


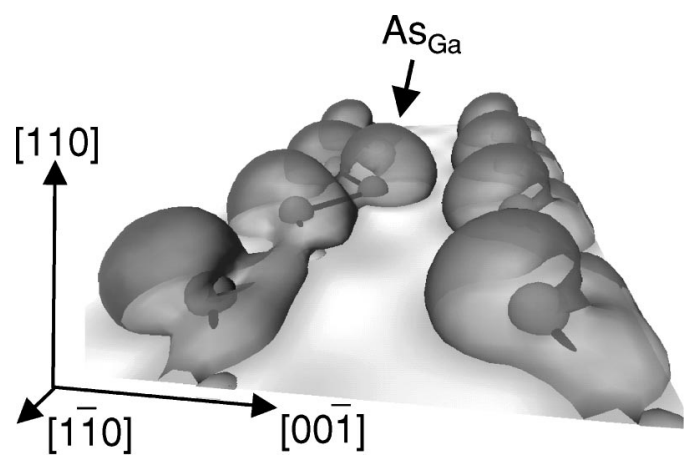

FIG. 4. Three-dimensional side view of the occupied states near the top of the valence band of $\mathrm{a} \mathrm{As}_{\mathrm{Ga}}$ in the surface layer is shown. The defect is localized in between the rows of occupied surface dangling bonds and exhibits a localized defect state directly above the arsenic atom on the $\mathrm{Ga}$ lattice site. Arsenic surface atoms are shown at the centers of the dangling bonds. The isosurface includes all occupied states between the VBM and $\mathrm{VBM}-0.7 \mathrm{eV}$ for a charge density of $5 \times 10^{-3} \AA^{-3}$.

recognizable: (i) The simulation shows the presence of an additional maximum in the occupied density of states [Fig. 2(c)] as observed experimentally [Figs. 2(a) and 3(a), and 3(c)]. (ii) The neighboring occupied dangling bonds change their shape at low energies close to the top of the valence band in a manner directly comparable to the STM images, i.e., the slight displacement and rotation of the orientation of the dangling bonds, which are typical for the surface anion antisite. (iii) We find in the simulation the same subtle displacement of one empty dangling bond [Fig. 2(d)] as observed in the STM images [Fig. 2(b)]. It is important to note that (ii) and (iii) are purely electronic effects and not related to the actual atomic coordinates. The atomic displacements around the defect are smaller than $0.1 \AA$ and the geometric shift of the antisite is in the opposite direction as compared to the displacement of the occupied dangling bond [see Figs. 2(b) and 2(d)].

From this discussion, we conclude that the STM images indeed show the surface anion antisite defect on GaAs, GaP, and InP surfaces. Note that the occupied defect state of the surface antisite defect is very localized in the experiment as well as in the calculation, in contrast to the subsurface or bulk antisite defects. ${ }^{1-3,16,17}$ The contrast of the subsurface $\mathrm{As}_{\mathrm{Ga}}$ antisite defects extends in STM images over three to four lattice spacings ${ }^{1}$ and has been identified as the tails of the defect states reaching the surface. ${ }^{3}$ In our case, however, the contrast in STM images of the surface antisite defect arises mainly from the presence of an occupied dangling bond at the As atom on the Ga lattice site. This is demonstrated in Fig. 4, which shows a three-dimensional side view of the occupied states near the VBM. The state in between the rows of occupied dangling bonds is the filled antisite related dangling bond, which causes the feature in the occupied state STM images. The facts that the feature is only strong at low voltages and apparently disappears at higher magnitudes of voltages are likely to be the reasons why the surface antisite defect has not been observed previously. Finally, we note that the antisite defects observed were not present in the bulk material, since we only observed the surface but no subsurface antisite defects. The defect formation mechanism is currently under investigation.

The authors thank K. H. Graf for excellent technical support and the Bundesministerium für Forschung und Technologie as well as the Deutsche Forschungsgemeinschaft and the Sonderforschungsbereich 296 for financial support.

${ }^{1}$ R. M. Feenstra, J. M. Woodall, and G. D. Pettit, Phys. Rev. Lett. 71, 1176 (1993).

${ }^{2}$ B. Grandidier, H. Chen, R. M. Feenstra, D. T. McInturff, P. W. Juodawlkis, and S. E. Ralph, Appl. Phys. Lett. 74, 1439 (1999).

${ }^{3}$ R. B. Capaz, K. Cho, and J. D. Joannopoulos, Phys. Rev. Lett. 75, 1811 (1995).

${ }^{4}$ R. M. Feenstra, J. A. Stroscio, J. Tersoff, and A. P. Fein, Phys. Rev. Lett. 58, 1192 (1987).

${ }^{5}$ Ph. Ebert, B. Engels, P. Richard, K. Schroeder, S. Blügel, C. Domke, M. Heinrich, and K. Urban, Phys. Rev. Lett. 77, 2997 (1996).

${ }^{6}$ R. J. Hamers, J. Vac. Sci. Technol. B 6, 1462 (1988).

${ }^{7}$ J. A. Stroscio, R. M. Feenstra, and A. P. Fein, Phys. Rev. Lett. 58, 1668 (1987).

${ }^{8}$ G. Schwarz, A. Kley, J. Neugebauer, and M. Scheffler, Phys. Rev. B 58, 1392 (1998)

${ }^{9}$ M. Bockstedte, A. Kley, J. Neugebauer, and M. Scheffler, Comput. Phys. Commun. 107, 187 (1997).

${ }^{10}$ J. Tersoff and D. R. Hamann, Phys. Rev. B 31, 805 (1985).

${ }^{11} \mathrm{Ph}$. Ebert, Surf. Sci. Rep. 33, 121 (1999).

${ }^{12}$ G. A. Baraff and M. Schlüter, Phys. Rev. Lett. 55, 1327 (1985).

${ }^{13}$ S. B. Zhang and J. E. Northrup, Phys. Rev. Lett. 67, 2339 (1991)

${ }^{14}$ J. Dabrowski and M. Scheffler, Phys. Rev. B 40, 10391 (1989).

${ }^{15} \mathrm{Ph}$. Ebert, K. Urban, L. Aballe, C. H. Chen, K. Horn, G. Schwarz, J. Neugebauer, and M. Scheffler, Phys. Rev. Lett. 84, 5816 (2000).

${ }^{16}$ N. D. Jäger and E. R. Weber, in Semiconductors and Semimetals, edited by M. Stavola (Academic, New York, 1998), Vol. 51B, p. 261.

${ }^{17}$ B. Grandidier, X. de la Broise, D. Stievenard, C. Delerue, M. Lannoo, M. Stellmacher, and J. Bourgoin, Appl. Phys. Lett. 76, 3142 (2000). 